\title{
Troubleshooting for Ureteroscopy Complicated by Unexpected Guidewire Looping and Entrapment
}

\author{
Ioannis Efthimiou, MD, MSc, FEBU, \\ Zacharias Chousianitis, MD, and Kostadinos Skrepetis, MD, PhD, FEBU
}

\begin{abstract}
This report presents a bizarre and unusual case of looping and entrapment of a hydrophilic guidewire in the ureter of a 65-year-old woman with a stone in the lower ureter at the time of ureteroscopy and laser lithotripsy. We describe endourology maneuvers to remove the retained guidewire. Looping and entrapment of guidewire is a rare complication that urologists may face when they do not follow the basic principles of guidewire insertion. In that case, successful resolution is based both on surgeon's inspiration to apply the appropriate endourology maneuvers and availability of a variety of endourology tools in the theater. Both may lead urologists to avoid more invasive and morbid approaches for their patients.
\end{abstract}

Keywords: guidewire, complication, endourology

\section{Introduction and Background}

$\mathbf{G}$ UIDEWIRES ARE STANDARD tools in the field of endourology, in interventional radiology, and in angiographic

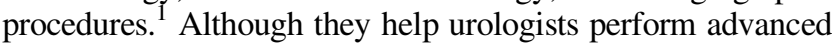
endourology procedures, they may become the source of potential complications such are perforation, bleeding, and kinking. ${ }^{2,3}$ Looping of guidewire is another poorly described and bizarre complication in the literature and is usually combined with knotting or kinking. ${ }^{2,3}$ However, looping and entrapment of a hydrophilic guidewire inside the ureter has never been reported in the literature. We report such a case and describe troubleshooting for this scenario.

\section{Presentation of Case}

A 65-year-old woman with an $8 \mathrm{~mm}$ stone in the right lower third of the ureter was admitted for rigid ureteroscopy and laser lithotripsy in our department. Under general anesthesia, the $21 \mathrm{~F}$ rigid cystoscope was inserted into the bladder and the distal part of a Double-J ureteral stent, which had been placed in a previous procedure, was retracted with a grasping forceps out of the bladder. A straight hydrophilic 0.035 " guidewire was advanced through the lumen of the stent into the ureter without real-time fluoroscopy. However, unexpectedly, during the advancement of the wire, the proximal part of it came out through the external urethral orifice (Fig. 1A) and fluoroscopy revealed looping back of the shaft of the guidewire below the stone (Fig. 1B). Initially, we tried unsuccessfully to retract it manually backwards, pulling sequentially each end of the guidewire. Next, we placed a second hydrophilic safety $0.035^{\prime \prime}$ guidewire up to the kidney under fluoroscopic guidance and we repeated the same maneuver without any success. Then, we inserted the semirigid Olympus WA29048A ureteroscope to inspect the ureter. The guidewire had perforated the mucosa of the ureter and was looping back through the lumen of the ureter (Fig. 2). Another attempt to remove the wire, keeping the ureteroscope stable inside the ureter, was also unsuccessful. The scope was removed and we advanced a standard $5 \mathrm{~F}$ open-end ureteral catheter over the guidewire up to the level of the loop, but once again, withdrawal of the guidewire was impossible. Finally, we repeated the previous step with an $8 \mathrm{~F}$ stiff, superglide ureteral catheter (Rüsch; Teleflex) (Fig. 3). The guidewire was removed easily from the ureter through the ureteral catheter without any sequela. Retrograde urography did not show any contrast extravasation, but the ureter was edematous and the vision was poor due to hemorrhage. So a 7F open-end Double-J ureteral stent was inserted fluoroscopically and the procedure was rescheduled to 4 weeks later.

Department of Urology, General Hospital of Messinia, Kalamata, Greece.

(C) Ioannis Efthimiou et al. 2017; Published by Mary Ann Liebert, Inc. This is an Open Access article distributed under the terms of the Creative Commons Attribution License, which permits unrestricted use, distribution, and reproduction in any medium, provided the original work is properly cited. 

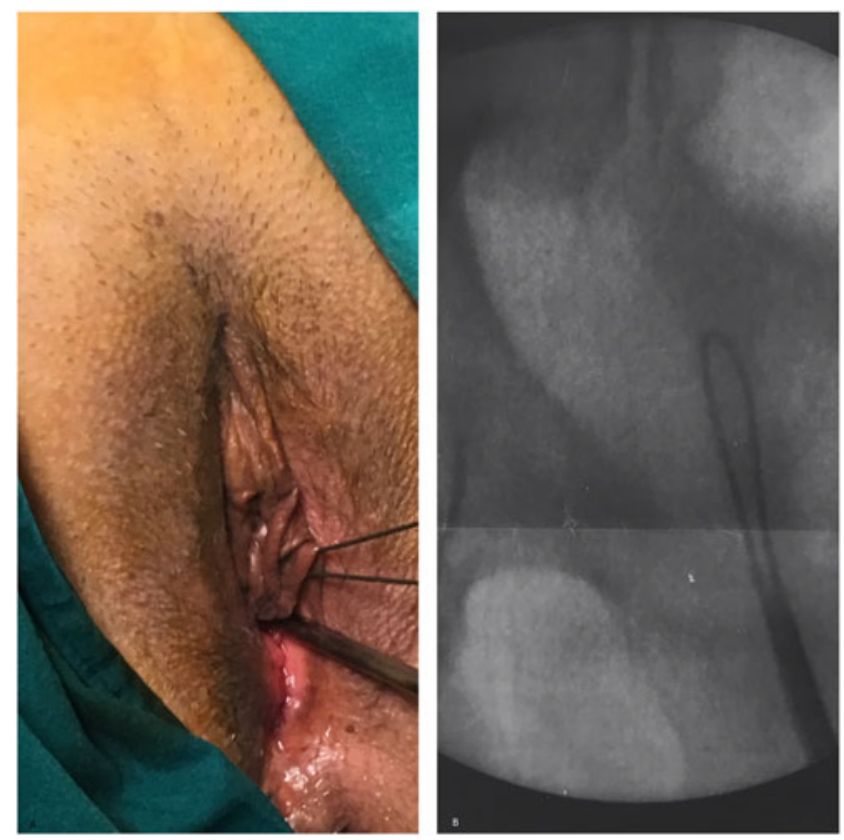

FIG. 1. (A) Direct view. Both parts of the guidewire protrude out of the urethra. (B) Fluoroscopy. Formed inverted "U" of the guidewire in the lower third of the ureter.

\section{Discussion}

When excessive length of a guidewire is forced in a confined space, this may lead in one of three scenarios. First, the extra length of guidewire forms a coil or loop within that space. This is commonly seen when the flexible tip of the guidewire cannot bypass a stop, for example, an impacted stone. Second, if the operator applies more force than the

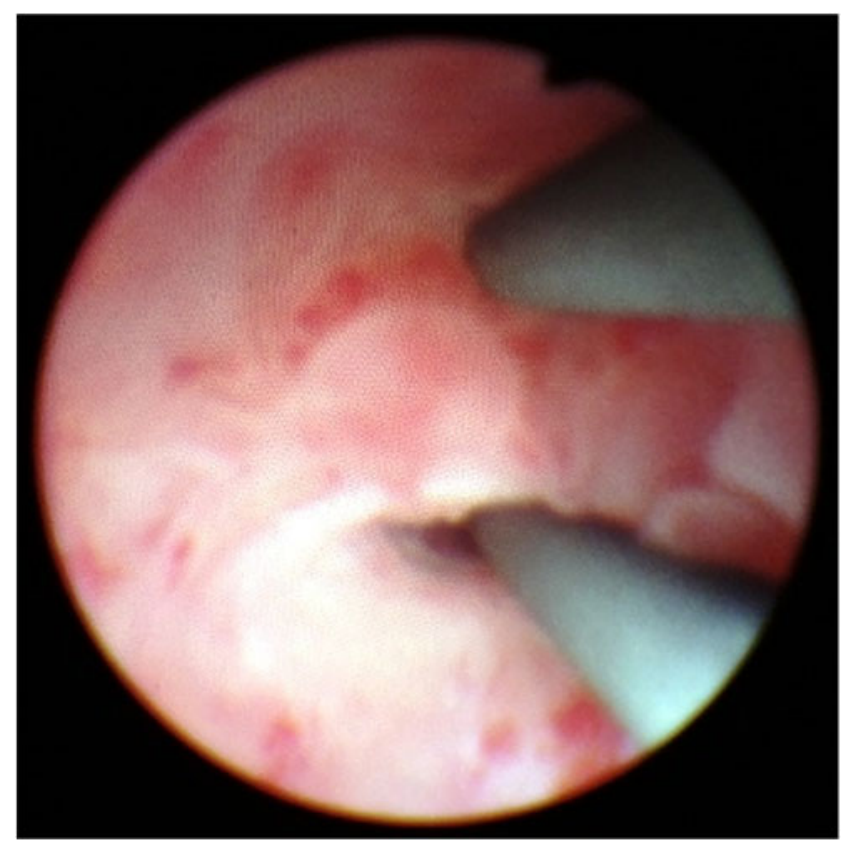

FIG. 2. Endoscopic view: The guidewire has initially passed submucosally and returned intraluminally, entrapping the mucosa between both ends.

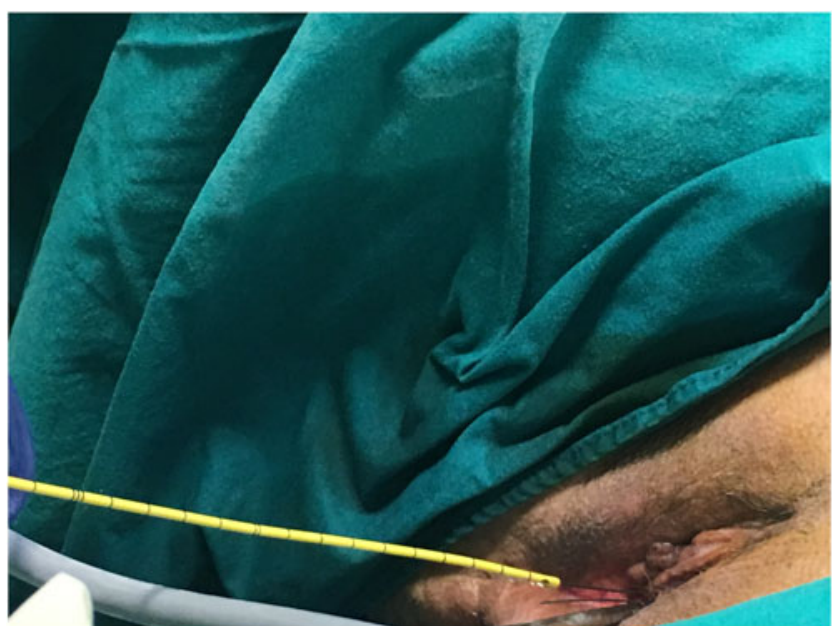

FIG. 3. A superglide ureteral catheter is advanced through the guidewire.

guidewire can resist, the loop may tighten within and eventually may form a kink or a knot. This is extremely unusual in the case of the ureter due to the configuration of the organ. However, cases of such complication have been described in the literature, especially during endovascular or interventional radiology procedures. ${ }^{1}$ Third, the tip of the guidewire may perforate the wall of the lumen and advance outside the organ.

In our case, the forced blindly guidewire formed an inverted "U," followed an opposite direction, came out the ureteral orifice, moved down to the bladder, inserted into the urethra, and finally came out of the urethral meatus without any kinking in its shaft.

A possible explanation for that event could be that the straight tip of the guidewire perforated initially the mucosa and after a few centimeters, it came again intraluminally where it met the resistance of the impacted stone and looped upon itself. Excessive force from the operator without fluoroscopy guidance led the wire to follow the opposite direction and come outside the urethra. The confined space in ureteral lumen, the increased radial force of the wire, and the perforation of the mucosa contributed to entrapment of the guidewire, although it was a hydrophilic one. Also, it is unclear if a manufacturing defect of the hydrophilic coating in the guidewire contributed to this event. The superglide stiff ureteral catheter decreased the friction because it was intervened between the wire and the mucosa and eased the withdrawal of it acting as a lever.

Looking back after having managed this issue, we believe that if the procedure was done properly and under strict fluoroscopic guidance from the beginning, even in the presence of a previously inserted Double-J ureteral stent, advancement and entrapment of the guidewire to this extent would have been avoided. According to basic principles of guidewire insertion, ${ }^{4}$ the motion of the guidewire should be monitored all throughout the procedure under direct control vis-à-vis or adjacent to the previously placed ureteral stent. Once the proximal coil of the wire is noted to be in the renal pelvis, the previously placed Double-J ureteral stent may be removed with a grasper leaving behind the hydrophilic wire, thereby eliminating the risk of losing the ureteral access. The previously inserted hydrophilic guidewire may then be 
exchanged with an Amplatz super stiff guidewire by inserting an open-ended ureteral catheter through it.

\section{Conclusion}

Looping and entrapment of guidewire is a rare complication that urologists may face when they do not follow the basic principles of guidewire insertion. In that case, successful resolution is based both on surgeon's inspiration to apply the appropriate endourology maneuvers and availability of a variety of endourology tools in the theater. Both may lead urologists to avoid more invasive and morbid approaches for their patients.

\section{Disclosure Statement}

No competing financial interests exist.

\section{References}

1. Wang HE, Sweeney TA. Subclavian central venous catheterization complicated by guidewire looping and entrapment. J Emerg Med 1999;17:721-724.

2. Kipling M, Mohammed A, Medding RN. Guidewires in clinical practice: Applications and troubleshooting. Expert Rev Med Devices 2009;6:187-195.
3. Tan GH, Ramasamy HK, Git KA. The Guide Wire: When too much of a good thing is no good at all. Uro Today Int 2012;5:65

4. Shah O, Hyams E. Access to the ureter: Flexible ureteroscopy. In: Smith A, Badlani G, Preminger G, Kavoussi L (eds.) Smith's Textbook of Endourology. UK: Wiley-Blackwell, 2012, pp. 402-411.

Address correspondence to: Ioannis Efthimiou, $M D, M S c, F E B U$

Department of Urology General Hospital of Messinia Megalou Alexandrou 130 TK 24132 Kalamata Greece

E-mail: efthimiou_ioannis@hotmail.com

Cite this article as: Efthimiou I, Chousianitis Z, Skrepetis K (2017) Troubleshooting for ureteroscopy complicated by unexpected guidewire looping and entrapment, Journal of Endourology Case Reports 3:1, 84-86, DOI: 10.1089/cren.2017.0052. 